\title{
Preparation of a Thermosensitive Guanidine-Chitosan/Poloxamer in situ Gel for Intranasal Delivery System
}

\author{
Ying-Zong Ren ${ }^{a}$, Xue-Jie Qi, Yuan-Lu Cui ${ }^{\text {b, }}$ \\ Tianjin State Key Laboratory of Modern Chinese Medicine, \\ Tianjin University of Traditional Chinese Medicine, Tianjin, 300193, China \\ a email: 15099660621ren@163.com \\ b,* Corresponding author email: ylcui@tjutcm.edu.cn
}

Keywords: Themosensitive hydrogel; Orthogonal Design; Guanidine-Chitosan; Poloxamer

\begin{abstract}
A novel themosensitive in situ gel was prepared by using Poloxamer407 and poloxamer188 combined with membrane penetration enhancer Guanidine-Chitosan. Orthogonal experiment optimization was used to study of formulations conditions that suit to intranasal drug delivery. The optimal intranasal gel formulation has been further checked for viscosity behavior against temperature. The result showed that the optimal formulation was 18\% P407 (w/w), 3\% P188 $(\mathrm{w} / \mathrm{w}), 2 \%$ GCS $(\mathrm{w} / \mathrm{w})$, which have prospective gelation temperature $29.5{ }^{\circ} \mathrm{C}$. Evaluation of Guanidine-chitosan influence in gelation temperature showed slight decrease the point of hydrogel system.
\end{abstract}

\section{Introduction}

Nasal route drug delivery due to good patient compliance and higher bioavailability for drugs makes it has been paid much attention in recent years. This route offers alternative methods over administration for systemically active drugs, especially for those that enzyme degradation in the gastrointestinal tract or undertake first pass metabolism liver [1, 2]. Poloxamers, the trade name Pluronics from BASF, are commercially available copolymers. Poloxamers have properties of change individual copolymer form self-assemble micelles or appearance gelling behavior to response temperature simulate in aqueous. This temperature responsive property makes it can wrap hydrophobic drugs turn to water soluble, thus improving target drugs solubility [3]. Themosensitive property of Pluronic F127 (Poloxamer P407) with the molecular weight of 12,600 is able to achieve strength gels in the temperature range of $30-50{ }^{\circ} \mathrm{C}$. [4] In aqueous, Poloxamers transform from sol to gel above the critical gelation temperature (CMT) and the critical gelation concentrations (CMC). It was reported that as the concentration increased the critical gelation temperature would decrease, and vice versa. The sol to gel phase transforming behavior of the pluronics in water is relative both with temperature and molecular weight. The additives have properties of enhance mucoadhesive and bioavailability of drugs in the nasal [5]. The guanidine derivatives can significantly enhance the positive charge density of the chitosan, which led easier to combine with the cell surface [6]. The purpose of this study was to explore a novel injectable thermosensitive hydrogel system for drug release by combining two kinds of Poloxamers (P407 and P188) and together with guanidine chitosan as additives [7].

\section{Material and method}

Poloxamers (P407, P188) were purchased from Sigma-Aldrich (USA). Chitosan was supplied from Shanghai Sangon Biotech Co., Ltd. (China). with 90\% deacetylation degree. Cyanamide (98\%) was obtained from Alfa Aesar (USA). All other chemicals and reagents used in the study were of analytical grade or ACS grade without any further purification process. Distilled water was used throughout all the experiments. 


\section{Preparation of Guanidinylated Chitosan (GCS) and Thermo-sensitive Hydrogel}

Poloxamers thermo-sensitive hydrogels were prepared by using the "cold method" reported by Bermudez [7], which is help for Poloxamers dissolving in aqueous and avoids possible alterations influenced by temperature. Simply, appropriate amounts of Poloxamers were directly added to the cold water $\left(0-4{ }^{\circ} \mathrm{C}\right)$ and kept on the ice bath while maintaining constant agitation with magnetic stirring rod. In order to keep fully swelling, Poloxamers solutions were put in a $4{ }^{\circ} \mathrm{C}$ refrigerator overnight. Chitosan was guanidinylated by the synthetic procedures given in the literature using cyanamide and hydrochloric acid solution (0.33M) [8]. Simply, a certain amount of chitosan (0.32 g) was mixed with hydrochloric acid solution at the weight ratio of 1:100. After an appropriate contents of cyanamide added to the mixture and then the solutions were kept at $90{ }^{\circ} \mathrm{C}$ under stirring for $12 \mathrm{~h}$. The result solution was dialyzed in distilled water (MW of 3500) for $3 \mathrm{~d}$ and lyophilized.

\section{Experiments design and measurement of gelation temperature}

The gelation temperature of the gel was tested by the intelligent temperature metal bath. A metal content bath thermometer was controlled temperature associated to a digital thermometer and kept vial filled with the Poloxamers hydrogel at a content temperature, with heating rate at any temperature from -10 to $105{ }^{\circ} \mathrm{C}$ [7]. The temperature of metal content thermometry was increased in increments of $1{ }^{\circ} \mathrm{C}$, and left to equilibrate $3 \mathrm{~min}$ at each new set. The temperature was determined as a critical gelation temperature at the point of meniscus would no longer move upon tilting through $90^{\circ}$ angle. In order to get an injectable drug delivery hydrogel, the liquid gelation temperature range from 30 to $37^{\circ} \mathrm{C}$, so as to be in a liquid form at room temperature and to form a gel phase instantly in the body without leakage. In order to test the critical temperature suits for human body, the gelation temperature optimization including P407 concentration, P188 concentration, and GCS concentration, for which those three factors are parallel single factor to study their effect on gelation temperature. Before the orthogonal test, the Single factors near the optimal conditions should be initially determined so as to look for the most approximately truly condition. For different P407 concentrations (18\%, 20\% and 22\%), P188 concentrations (1\%, 2\% and $3 \%)$ and GCS concentrations (1\%, $2 \%, 3 \%$ and $4 \%)$, respectively, parallel single factor experiments were carried out. The single factor experiments responsive values were shown in Fig.1. According to the single factor tests, the L9 $\left(3^{4}\right)$ was designed to further study optimal formula conditions, the factors and levels were showed in Table 1 . The intuitive and variance analysis were used to calculate the results.

Table 1. Levels and factors of orthogonal experiments design

\begin{tabular}{cccc}
\hline Level & A & B & C \\
& Concentration of P407 (\%) & Concentration of P 188 (\%) & Concentration of GCS (\%) \\
\hline 1 & 18 & 2 & 1 \\
2 & 20 & 3 & 2 \\
3 & 22 & 4 & 3 \\
\hline
\end{tabular}

\section{Results}

Numerous approaches have been investigated to improve the bioavailability of drugs. Several kinds of biomaterials were used to enhance cell membrane permeability. In this study, GCS not only improved cell membrane permeability but also modulated P407 gelation temperature. The results responsive values of the single factors tests and orthogonal experiments results showed in Table2. The intuitive analysis depicted in Table 2, the influence of the P407 concentration on gelation temperature was the most important. As the range of data $\mathrm{R}$ shown, the effect order of kinds factors on the gelation temperature was: P407 > GCS > P188, the optimum formula was: $\mathrm{A}_{2} \mathrm{~B}_{3} \mathrm{C}_{2}$, namely 18\% P407 (w/w), 3\% P188 (w/w), 2\% GCS (w/w). The results of viscosity of hydrogel against temperature showed the data of viscosity increase with the temperature rise. It is hinted that the hydrogel transformed from sol to gel. The results of variance analysis were showed in Table3, all components in the formulas were significant influencing factor of gelling temperature. 


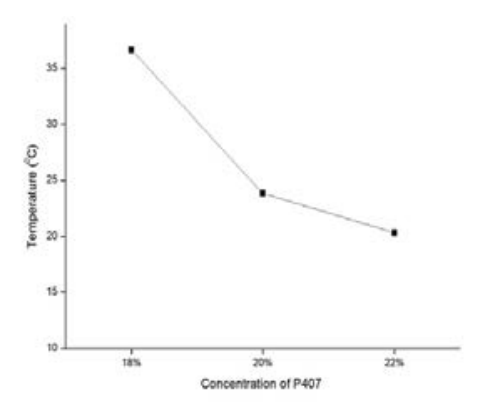

(a) Concentration of P407

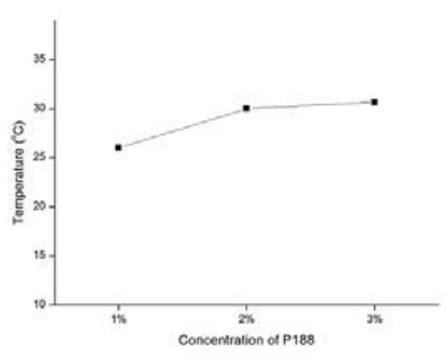

(b) Concentration of P188

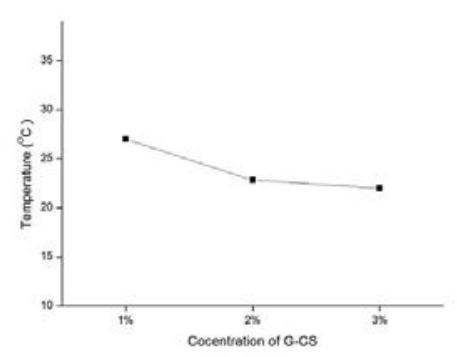

(c) Concentration of GCS

Fig.1. Effects of various factors on GCS- P407 / P188 themosensitive hydrogel

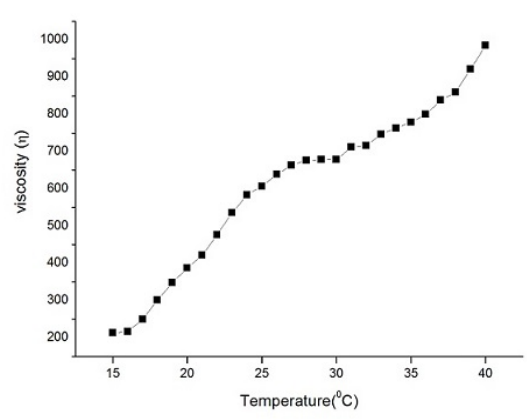

Fig.2. Dynamic viscosity change with temperature rise in optimal formula of GCS-P407 / P188.

Table 2. Orthogonal experimental design and responsive values $(n=3)$

\begin{tabular}{cccccc}
\hline & Error & P407 & P188 & GCS & average \\
\hline A1 & 1.0 & 0.48 & 0.06 & 0.03 & 35.8 \\
B2 & 1.0 & 0.54 & 0.09 & 0.06 & 30.2 \\
C3 & 1.0 & 0.60 & 0.12 & 0.09 & 22.0 \\
D4 & 2.0 & 0.48 & 0.09 & 0.09 & 37.5 \\
E5 & 2.0 & 0.54 & 0.12 & 0.03 & 33.1 \\
F6 & 2.0 & 0.60 & 0.06 & 0.06 & 23.0 \\
G7 & 3.0 & 0.48 & 0.12 & 0.06 & 39.0 \\
H8 & 3.0 & 0.54 & 0.06 & 0.09 & 30.4 \\
I9 & 3.0 & 0.60 & 0.09 & 0.03 & 26.4 \\
K1 & 88.0 & 112.3 & 89.2 & 95.3 & \\
K2 & 93.6 & 93.7 & 94.1 & 92.2 & \\
K3 & 95.7 & 71.4 & 94.07 & 89.9 & \\
k1 & & 37.4 & 29.7 & 31.8 & \\
k2 & & 31.2 & 31.4 & 30.7 & \\
k3 & & 23.8 & 31.4 & 30.0 & \\
R & & 13.6 & 1.6 & 1.8 & \\
\hline
\end{tabular}


Table 3. Analysis of variation

\begin{tabular}{llllll}
\hline Source of variation & SS & df & MS & F & Significant \\
\hline 1.000 & 723.500 & 2.000 & 361.750 & 391.081 & $*$ \\
2.000 & 146.000 & 2.000 & 73.000 & 78.919 & $*$ \\
3.000 & 42.000 & 2.000 & 21.000 & 22.703 & $*$ \\
4.000 & 18.500 & 2.000 & 9.250 & 10.000 & $*$ \\
SS $_{\mathrm{T}}$ & 0.000 & 18.000 & & & \\
\hline \multicolumn{5}{c}{ Note: “*” and “**” stand for significant at $P<0.05$ and $P<0.01$, respectively. }
\end{tabular}

\section{Conclusion}

In this study, the formula of the GCS-P407 / P188 system temperature sensitive in situ gel was selected by viscosity and tilt vials, and the optimal formula was achieved to be $18 \%$ P407 (w/w), $3 \% \mathrm{P} 188(\mathrm{w} / \mathrm{w}), 2 \% \mathrm{GCS}(\mathrm{w} / \mathrm{w})$ and the gelation temperature was $29.5^{\circ} \mathrm{C}$. The optimal formula was suitable to use as intranasal drug delivery system.

\section{Acknowledgement}

This work was supported by the National Natural Science Foundation of China (No. 81473542) and the Specialized Research Fund for the Doctoral Program of Higher Education (No. 20131210110008).

\section{References}

[1] Ravi P, Aditya N, Patil S, Cherian L. Nasal in-situ gels for delivery of rasagiline mesylate: improvement in bioavailability and brain localization[J]. Drug delivery 2013:1-8.

[2] Amidi M, Romeijn SG, Borchard G, Junginger HE, Hennink WE, Jiskoot W. Preparation and characterization of protein-loaded $\mathrm{N}$-trimethyl chitosan nanoparticles as nasal delivery system[J]. Journal of Controlled Release 2006;111:107-16.

[3] Kalshetti PP, Rajendra VB, Dixit DN, Parekh PP. Hydrogels as a drug delivery system and applications: a review[J]. Int J Pharm Pharm Sci 2012;4:1-7.

[4] Bonacucina G, Cespi M, Mencarelli G, Giorgioni G, Palmieri GF. Thermosensitive Self-Assembling Block Copolymers as Drug Delivery Systems[J]. Polymers 2011;3:779-811.

[5] Majithiya RJ, Ghosh PK, Umrethia ML, Murthy RS. Thermoreversible-mucoadhesive gel for nasal delivery of sumatriptan[J]. AAPS PharmSciTech 2006;7:E80-E6.

[6] Vinsova J, Vavrikova E. Recent advances in drugs and prodrugs design of chitosan[J]. Current pharmaceutical design 2008;14:1311-26.

[7] Bermudez J, Grau R. Thermosensitive poloxamer-based injectables as controlled drug release platforms for veterinary use: Development and in-vitro evaluation[J]. Int Res J Pharm Pharmacol 2011;1:109-18.

[8] Zhai X, Sun P, Luo Y, Ma C, Xu J, Liu W. Guanidinylation: A simple way to fabricate cell penetrating peptide analogue-modified chitosan vector for enhanced gene delivery[J]. Journal of Applied Polymer Science 2011;121:3569-78. 